\title{
Destroy to create: E3 ubiquitin ligases in neurogenesis Judith Stegmüller ${ }^{1 *}$ and Azad Bonni ${ }^{2}$
}

\author{
Addresses: ${ }^{1}$ Max-Planck-Institute of Experimental Medicine, Hermann Rein Strasse 3, 37075 Göttingen, Germany; ${ }^{2}$ Department of Pathology, \\ Harvard Medical School, 77 Ave Louis Pasteur, Boston, MA 02115, USA \\ *Corresponding author: Judith Stegmüller (stegmueller@em.mpg.de) \\ Fl000 Biology Reports 2010, 2:38 (doi:I0.34I0/B2-38)
}

The electronic version of this article is the complete one and can be found at: http://fl000.com/reports/biology/content/2/38

\begin{abstract}
The ubiquitin proteasome system (UPS) has drawn tremendous attention in the field of neuroscience. In recent years, we have gained insights into UPS-dependent mechanisms in brain development and disease. Several interesting studies over the past two years have highlighted the role of distinct E3 ubiquitin ligases in neurogenesis. Here, we will review the major findings in these studies and discuss their implications.
\end{abstract}

\section{Introduction and context}

The major function of the ubiquitin proteasome system (UPS) is to provide the regulated degradation of proteins [1]. The UPS consists of sets of enzymes that mark a protein destined for degradation with a polyubiquitin chain. In an enzymatic cascade, ubiquitin, which is a small $8.5-\mathrm{kDa}$ protein, is first covalently linked to the E1 ubiquitin-activating enzyme in an energy-dependent manner. Subsequently, ubiquitin is transferred onto the E2 ubiquitin-conjugating enzyme. Finally, the E3 ubiquitin ligase recruits both the E2 ubiquitin-conjugating enzyme and substrate, thereby facilitating the transfer of ubiquitin to the substrate. The processivity of the ubiquitination reaction leads to the formation of a polyubiquitin chain. Polyubiquitinated substrates are recognized by the proteasome, leading to the degradation of the substrate. While there are two characterized E1 enzymes and approximately 35 E2 enzymes, E3 ligases are quite numerous, with more than 600 enzymes [2]. Although E3 ligases confer substrate specificity, individual E3 ligases can recruit more than one substrate. Conversely, substrates may be targeted by more than one E3 ligase [3,4]. Among E3 ligases, RING (really interesting new gene)-type ligases represent the largest group and are composed of a single subunit or multisubunit complexes [2]. The RING-type E3 ligases operate as scaffolds to recruit both the $\mathrm{E} 2$ and the substrate to facilitate ubiquitination.
In contrast, members of the smaller family of HECT (homologues to E6AP C-terminus) ligases harbor enzymatic activity, which mediates the transfer of ubiquitin to the target [5]. Besides the control of protein abundance, ubiquitination regulates diverse aspects of cellular signaling and physiology $[6,7]$.

Over the past few years, a number of E3 ubiquitin ligases have been reported to play critical roles in diverse aspects of neuronal development, including axon, dendrite, and synapse morphogenesis (for reviews, see [8-10]). In this review, we will focus on recent studies implicating E3 ubiquitin ligases in the regulation of neurogenesis (Figure 1).

\section{Major recent advances}

E3 ubiquitin ligase pathways regulating neurogenesis

Neurogenesis requires the precise and sophisticated orchestration of many signaling events [11]. One way of determining the cell fate is to epigenetically program the genome to restrict the expression of genes that favor one cell fate over another. In this regard, a prominent factor is the repressor element 1 (RE-1)-silencing transcription factor (REST), also known as NRSF (neuron-restrictive silencing factor), which is a DNAbinding protein that recognizes a conserved motif present in many neuronal genes $[12,13]$. To ensure permanent gene silencing, REST associates with the 
Figure I. E3 ubiquitin ligases in neurogenesis

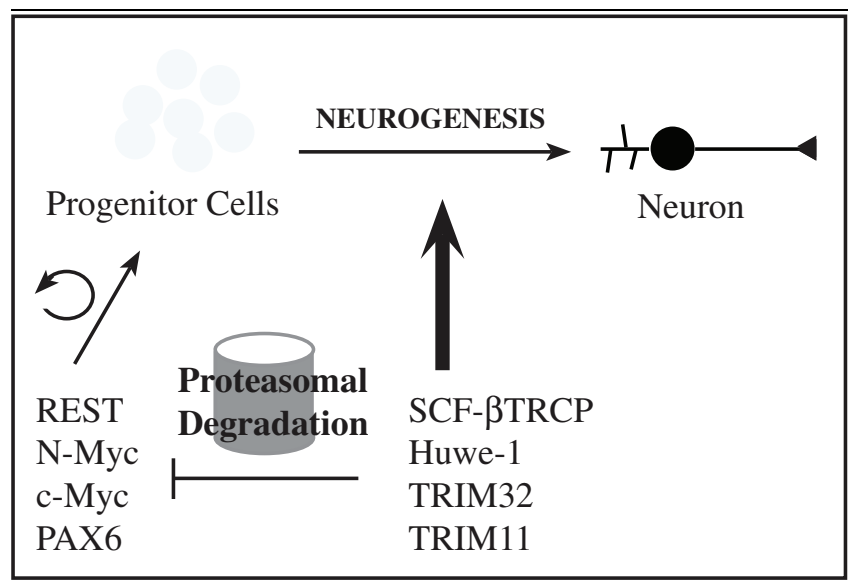

The E3 ubiquitin ligases SCF-3TRCP, Huwe-I, TRIM32, or TRIMI I promote neurogenesis by targeting their respective substrates REST, N-Myc, c-Myc, or PAX6 for degradation by the proteasome. $\beta$ TRCP, beta-transducin repeat containing protein; REST, repressor element I-silencing transcription factor; SCF; Skp, Cullin, F-box containing; TRIM, tripartite motif.

co-repressors mSin 3 and CoREST, which in turn recruit HDACs (histone deacetylases) to target genes [12]. The REST-dependent silencing mechanism is found in all non-neuronal cells, including those within the nervous system, raising the question of how precursor cells escape this silencing program to acquire a neuronal cell fate.

A recent study by Westbrook and colleagues [14] provides insight into this problem. The authors found that REST is polyubiquitinated and hence subjected to proteasomal degradation in embryonic stem (ES) cells. The authors then identified $\mathrm{SCF}^{\beta T R C P}$ as the $\mathrm{E} 3$ ubiquitin ligase that targets REST for degradation. SCF ${ }^{\beta T R C P}$ is an SCF complex consisting of the adaptor proteins $\underline{S} k p 1$ and $\underline{\text { Cullin } 1, \text { the }}$ RING component Roc1, and the F-box protein $\beta$ TRCP (beta-transducin repeat containing protein), which confers substrate specificity [15]. The recruitment of most F-box proteins, including $\beta$ TRCP, requires a phosphorylated recognition motif within the substrate [15]. Correspondingly, REST is recruited to $\operatorname{SCF}^{\beta T R C P}$ in a phosphodegron-dependent manner. To test the effect of the REST/SCF ${ }^{\beta T R C P}$ interaction on neuronal gene expression, ES cells harboring a Sox1 reporter gene as a marker of early-stage neurogenesis were transfected with REST, SCF $^{\text {BTRCP }}$, or control short interfering RNAs (siRNAs). Knockdown of $\beta$ TRCP led to significantly fewer Sox1positive cells as compared with control cells. In contrast, knockdown of REST strongly induced Sox1 expression. A comparably strong induction of Sox 1 expression was obtained by simultaneous knockdown of REST and BTRCP. In other experiments, ES cells containing the Sox1 reporter were transfected with wild-type or degron- mutant REST and placed in differentiation media. Expression of mutant REST, but not wild-type REST, dramatically reduced Sox1 expression. Taken together, these findings identify REST as a novel target of the E3 ubiquitin ligase $\mathrm{SCF}^{\beta T R C P}$ and support the conclusion that $\mathrm{SCF}^{\beta \mathrm{TRCP}}$ acts upstream of REST to control neurogenesis. While REST imposes a non-neuronal cell fate at an epigenetic level, controlled clearance of REST favors a neuronal cell fate by derepression and subsequent induction of neuronal genes.

The HECT domain E3 ubiquitin ligase Huwe1 employs a strategy similar to that of $\mathrm{SCF}^{\beta T R C P}$ to promote neurogenesis. The transcription factor N-Myc maintains the proliferation of precursor cells in the nervous system $[16,17]$. Previous studies have revealed that N-Myc undergoes proteasome-dependent degradation $[18,19]$. In an effort to determine how N-Myc is regulated, Lasorella and colleagues [20] used an unbiased biochemical approach to identify proteins that form a physical complex with N-Myc. The authors identified the E3 ubiquitin ligase Huwe1 as a new N-Myc-interacting protein. Huwe1 triggered the polyubiquitination and subsequent degradation of N-Myc [20]. To determine the functional consequence of Huwe1-mediated degradation of N-Myc in ES cells, wild-type and Huwe1 knockout ES cells were placed in differentiating conditions. While differentiated wild-type ES cells expressed neuronal genes, including neurogenin, $n$-cadherin, and MAP2, Huwe1 knockout ES cells failed to express these markers. Furthermore, knockout of Huwe1 in ES cells led to the accumulation of N-Myc protein. In other experiments, ex utero electroporation analyses of the cerebral cortex revealed that knockdown of N-Myc significantly decreased the number of proliferating cells and increased the number of neurons, suggesting that N-Myc knockdown leads to cell cycle exit of precursor cells. In contrast, knockdown of Huwe1 restored the proliferative nature of the cells and prevented differentiation along the neuronal lineage. In epistasis analyses, knockdown of N-Myc suppressed the Huwe1 knockdown-induced phenotype of increased cell proliferation and impaired differentiation, supporting the conclusion that N-Myc acts downstream of Huwe1 in precursor cells in the cerebral cortex.

In a follow-up study, Zhao and colleagues [21] reported conditional knockout of Huwe1 in the cerebral cortex of mice. The authors also provided evidence suggesting that the gene encoding the Notch ligand Delta-like 3 (Dll3) may act as a target of N-Myc. Accordingly, Dll3 levels are elevated in Huwe1-null brains as compared with wildtype brain. By silencing N-Myc and Dll3 in Huwe1 knockout animals, the loss-of-Huwe1 phenotype of hyperproliferation and defective differentiation was reversed. Huwe1 ensures a low level of N-Myc at 
a critical time point of development, when neural precursors are scheduled to differentiate. Destruction of $\mathrm{N}-\mathrm{Myc}$ results in the suppression of the dll3 gene. The authors reasoned that inactivation of the Dll3-notch pathway is critical for neural precursors to acquire a neuronal cell fate and that Huwe1 acts in a critical developmental window to target N-Myc for degradation and to induce neurogenesis.

Prior to acquiring a neuronal cell fate, the multipotential neural precursor cell undergoes asymmetric cell division, giving rise to a multipotential precursor cell and a restricted neuronal progenitor cell [22]. The latter inherits cell fate-determining proteins that initiate the production of neurons [22]. An asymmetrically distributed determinant is the ubiquitin ligase TRIM32 (tripartite motif-containing 32) [23,24]. TRIM32 is a member of the tripartite interaction motif family of single-subunit E3 ligases. These proteins typically harbor a RING domain, a B-box domain (BB), and a coiled-coil domain (CC). The family consists of at least 65 members, which are implicated in diverse cellular functions and pathological conditions [25]. The Drosophila orthologue of TRIM32, Brat, has been identified as a determinant of cell fate. Once inherited by the daughter cell, Brat triggers neuronal differentiation of Drosophila neuroblasts [23]. In addition, Drosophila Brat has been identified as an interaction partner of the RNAase Argonaute 1 (Ago1) [26]. Also, the transcription factor $\mathrm{dMyc}$ was found to be regulated post-transcriptionally downstream of Brat. The exact regulatory mechanism, however, remained unexplored until recently [23].

Schwamborn and colleagues [24] investigated TRIM32 function in the mammalian cerebral cortex. TRIM32 displayed an asymmetrical distribution in dividing precursor cells and was enriched in one of the two daughter cells [24]. In overexpression analyses of precursor cells, TRIM32 potently induced neuronal cell fate as monitored by neuron-specific class III $\beta$-tubulin expression. In addition to the conserved proneuronal function of TRIM32, the binding to Ago1 is conserved across species. Interestingly, the TRIM32/Ago 1 complex was found to bind to and activate the microRNA (miRNA) let-7a, which promoted the expression of class III $\beta$-tubulin in precursor cells. This proneural function of TRIM32, however, is independent of its ligase activity. To establish a functional relationship of TRIM32 and c-Myc based on the authors' previous findings [23], TRIM32 was subjected to immunoprecipitation analyses and found to interact with c-Myc. In addition, the RING domain of TRIM32 was required for the polyubiquitination and consequent degradation of c-Myc. Importantly, the degradation of c-Myc triggered cell cycle exit of mitotic cells, and expression of RINGdefective TRIM32 or TRIM32 together with c-Myc failed to induce neuronal differentiation in the cerebral cortex. Hence, the authors proposed a model in which TRIM32 triggers at least two events that promote neurogenesis: TRIM32 activates miRNAs and targets c-Myc for proteasomal degradation. Thus, the degradation of Myc family members appears to be a recurring concept in cells that differentiate along the neuronal lineage.

TRIM11 represents another member of the TRIM family that has been implicated in the regulation of neurogenesis. Tuoc and Stoykova [27] recently reported that TRIM11 may regulate the abundance of the transcription factor Pax6 in neural precursor cells. TRIM11 binds to and polyubiquitinates Pax6, thus triggering its degradation by the proteasome. Intriguingly, TRIM11 clears the cell of insoluble Pax6. Overexpression of TRIM11 in cortical precursor cells inhibits neuronal differentiation. Using siRNAs to silence TRIM11, the authors showed that TRIM11 knockdown led to the accumulation of Pax6 in cortical precursor cells, leading to apoptosis. While TRIM11 gain-of-function impaired neurogenesis, TRIM lossof-function induced apoptosis. Interestingly, TRIM11 itself is a Pax6-controlled gene, providing an elegant feedback mechanism of substrate and E3 ligase [27]. In summary, Tuoc and Stoykova [27] present a model in which TRIM11 regulates Pax6 levels to ensure the correct gene dosage of Pax6-controlled genes to promote neurogenesis.

\section{Future directions}

The featured studies in this review explore the role of the UPS in neurogenesis. Future studies in this area should provide further insights into the role of ubiquitin ligases in neurogenesis. The physiologic role of each of the E3 ubiquitin ligases in the development and function of the nervous system remains to be elucidated. In addition, how each of the $\mathrm{E} 3$ ubiquitin ligases, $\mathrm{SCF}^{\beta \mathrm{TRCP}}$, Huwe1, TRIM32, and TRIM11, is regulated in neural precursor cells will be a fruitful avenue for future research. Finally, it will be interesting to determine whether and how these distinct ubiquitin signaling pathways might interact with each other in neural stem cells and in the course of neuronal differentiation.

In view of the large number of E3 ubiquitin ligases, we are just beginning to gain an understanding of the role of these enzymes in the early events of neuronal development. Recent studies have implicated the major cell cycle ubiquitin ligases Cdh1-anaphase-promoting complex (Cdh1-APC) and Cdc20-APC in distinct aspects of neuronal development $[9,10,28]$. In particular, Cdh1APC has been implicated in the control of axon growth and patterning, whereas Cdc20-APC has been implicated 
in the control of dendrite morphogenesis and presynaptic differentiation in the mammalian brain [29-33]. Cdh1-APC has also been implicated in the control of synapse development in invertebrates $[34,35]$. Recent studies have broadened the roles of Cdh1-APC beyond development to the control of glycolysis and synapse function in the mature nervous system [36-38]. Interestingly, the roles of Cdh1-APC and Cdc20-APC in neurogenesis in the mammalian brain have yet to be elucidated. Since Cdh1-APC and Cdc20-APC are critical regulators of cell cycle progression, these ligases are anticipated to have important functions in the early events of neuronal development.

Beyond the APC, it will be interesting to explore the role of additional E3 ubiquitin ligases in neurogenesis. Screens might be a useful approach to identify novel E3 ligases that regulate neurogenesis. Since deregulation of mechanisms of neurogenesis is thought to play a critical role in disease pathogenesis [39], the identification of ubiquitin ligase pathways that control neurogenesis will advance our understanding of brain development and diseases. These studies also raise the prospect that therapies that target E3 ligases in order to stimulate neurogenesis in the adult brain may be developed. It will be an exciting and worthwhile endeavor to shed light on the complexity of E3 ubiquitin ligase function in neurogenesis.

\section{Abbreviations}

Ago1, Argonaute 1; APC, anaphase-promoting complex; BTRCP, beta-transducin repeat containing protein; Cdc20-APC, Cdc20-anaphase-promoting complex; Cdh1-APC, Cdh1-anaphase-promoting complex; Dll3, Delta-like 3; ES, embryonic stem; HECT, homologues to E6AP C-terminus; miRNA, microRNA; REST, repressor element 1-silencing transcription factor; RING, really interesting new gene; SCF complex; Skp, Cullin, F-box containing complex; siRNA, short interfering RNA; TRIM, tripartite motif; UPS, ubiquitin proteasome system.

\section{Competing interests}

The authors declare that they have no competing interests.

\section{Acknowledgments}

This work was supported by the Max Planck Society and the Deutsche Forschungsgemeinschaft (German Research Foundation) (JS) and by National Institutes of Health grants NS051255 and NS041021 (AB).

\section{References}

I. Hershko A, Ciechanover A: The ubiquitin system. Annu Rev Biochem 1998, 67:425-79.
2. Deshaies RJ, Joazeiro CA: RING domain E3 ubiquitin ligases. Annu Rev Biochem 2009, 78:399-434.

3. Nishitani H, Sugimoto N, Roukos V, Nakanishi Y, Saijo M, Obuse C, Tsurimoto T, Nakayama KI, Nakayama K, Fujita M, Lygerou Z, Nishimoto T: Two E3 ubiquitin ligases, SCF-Skp2 and DDBICul4, target human CdtI for proteolysis. EMBO J 2006, 25: 1126-36.

4. Amati B: Myc degradation: dancing with ubiquitin ligases. Proc Natl Acad Sci U S A 2004, I0 I:8843-4.

5. Rotin D, Kumar S: Physiological functions of the HECT family of ubiquitin ligases. Nat Rev Mol Cell Biol 2009, 10:398-409.

6. Wang C, Deng L, Hong M, Akkaraju GR, Inoue J, Chen ZJ: TAKI is a ubiquitin-dependent kinase of MKK and IKK. Nature 200I, 4I 2:346-5 I.

FI000 Factor 3.2 Recommended Evaluated by Tom Rapoport 19 Apr 2002, Anjana Rao 09 Oct 2001

7. Sun $L$, Chen ZJ: The novel functions of ubiquitination in signaling. Curr Opin Cell Biol 2004, 16:1 19-26.

8. Yi JJ, Ehlers MD: Emerging roles for ubiquitin and protein degradation in neuronal function. Pharmacol Rev 2007, 59:14-39.

9. Stegmuller J, Bonni A: Moving past proliferation: new roles for Cdh I-APC in postmitotic neurons. Trends Neurosci 2005, 28:596601 .

10. Yang $Y$, Kim AH, Bonni $A$ : The dynamic ubiquitin ligase duo: CdhI-APC and Cdc20-APC regulate neuronal morphogenesis and connectivity. Curr Opin Neurobiol 2010, 20:92-9.

II. Gotz M, Huttner WB: The cell biology of neurogenesis. Nat Rev Mol Cell Biol 2005, 6:777-88.

12. Ballas N, Mandel G: The many faces of REST oversee epigenetic programming of neuronal genes. Curr Opin Neurobiol 2005, 15:500-6.

13. Ooi L, Wood IC: Chromatin crosstalk in development and disease: lessons from REST. Nat Rev Genet 2007, 8:544-54.

14. Westbrook TF, Hu G, Ang XL, Mulligan P, Pavlova NN, Liang A, Leng Y, Maehr R, Shi Y, Harper JW, Elledge SJ: SCFbeta-TRCP controls oncogenic transformation and neural differentiation through REST degradation. Nature 2008, 452:370-4.

FI000 Factor 3.0 Recommended Evaluated by Deyou Zheng 26 Mar 2008

15. Ang XL, Harper JW: Interwoven ubiquitination oscillators and control of cell cycle transitions. Sci STKE 2004, 2004:pe3I.

16. Singh AM, Dalton S: The cell cycle and Myc intersect with mechanisms that regulate pluripotency and reprogramming. Cell Stem Cell 2009, 5:141-9.

17. Patel JH, Loboda AP, Showe MK, Showe LC, McMahon SB: Analysis of genomic targets reveals complex functions of MYC. Nat Rev Cancer 2004, 4:562-8.

18. Ciechanover A, DiGiuseppe JA, Bercovich B, Orian A, Richter JD, Schwartz AL, Brodeur GM: Degradation of nuclear oncoproteins by the ubiquitin system in vitro. Proc Natl Acad Sci U S A I991, 88: 139-43.

19. Gross-Mesilaty S, Reinstein E, Bercovich B, Tobias KE, Schwartz AL, Kahana C, Ciechanover A: Basal and human papillomavirus E6 oncoprotein-induced degradation of Myc proteins by the ubiquitin pathway. Proc Natl Acad Sci U S A 1998, 95:8058-63.

20. Zhao X, Heng Jl, Guardavaccaro D, Jiang R, Pagano M, Guillemot F, lavarone $A$, Lasorella $A$ : The HECT-domain ubiquitin ligase Huwel controls neural differentiation and proliferation by destabilizing the N-Myc oncoprotein. Nat Cell Biol 2008, I0: 643-53.

21. Zhao X, D'Arca D, Lim WK, Brahmachary M, Carro MS, Ludwig T, Cardo CC, Guillemot F, Aldape K, Califano A, lavarone A, Lasorella A: The N-Myc-DLL3 cascade is suppressed by the ubiquitin ligase Huwe I to inhibit proliferation and promote neurogenesis in the developing brain. Dev Cell 2009, 17:210-21. 
22. Zhong $W$, Chia $W$ : Neurogenesis and asymmetric cell division. Curr Opin Neurobiol 2008, I 8:4-II.

23. Betschinger J, Mechtler K, Knoblich JA: Asymmetric segregation of the tumor suppressor brat regulates self-renewal in Drosophila neural stem cells. Cell 2006, I 24: |24|-53.

FI000 Factor 7.0 Must Read

Evaluated by Valera Vasioukhin 30 Mar 2006, Orly Reiner 03 Apr 2006, Sally Temple 06 Apr 2006, Talila Volk 24 Apr 2006, Andreas Wodarz 02 May 2006

24. Schwamborn JC, Berezikov E, Knoblich JA: The TRIM-NHL protein TRIM32 activates microRNAs and prevents selfrenewal in mouse neural progenitors. Cell 2009, 136:9|3-25.

FI000 Factor 6.0 Must Read

Evaluated by Orly Reiner 20 Mar 2009

25. Meroni G, Diez-Roux G: TRIM/RBCC, a novel class of 'single protein RING finger' E3 ubiquitin ligases. Bioessays 2005, 27: | | 47-57.

26. Neumuller RA, Betschinger J, Fischer A, Bushati N, Poernbacher I, Mechtler K, Cohen SM, Knoblich JA: Mei-P26 regulates microRNAs and cell growth in the Drosophila ovarian stem cell lineage. Nature 2008, 454:24I-5.

27. Tuoc TC, Stoykova A: TrimII modulates the function of neurogenic transcription factor Pax6 through ubiquitinproteosome system. Genes Dev 2008, 22:1972-86.

28. Kim $A H$, Bonni $A$ : Thinking within the $\mathbf{D}$ box: initial identification of Cdh I-APC substrates in the nervous system. Mol Cell Neurosci 2007, 34:28I-7.

29. Konishi Y, Stegmuller J, Matsuda T, Bonni S, Bonni A: CdhI-APC controls axonal growth and patterning in the mammalian brain. Science 2004, 303:1026-30.

FI000 Factor 6.0 Must Read

Evaluated by Genevieve Rougon 19 Feb 2004

30. Stegmuller J, Konishi Y, Huynh MA, Yuan Z, Dibacco S, Bonni A: Cellintrinsic regulation of axonal morphogenesis by the CdhIAPC target SnoN. Neuron 2006, 50:389-400.

31. Lasorella A, Stegmuller J, Guardavaccaro D, Liu G, Carro MS, Rothschild G, de la Torre-Ubieta L, Pagano M, Bonni A, lavarone A: Degradation of Id2 by the anaphase-promoting complex couples cell cycle exit and axonal growth. Nature 2006, 442:47I-4.
32. Kim AH, Puram SV, Bilimoria PM, Ikeuchi Y, Keough S, Wong M, Rowitch D, Bonni A: A centrosomal Cdc20-APC pathway controls dendrite morphogenesis in postmitotic neurons. Cell 2009, 136:322-36.

FI000 Factor 6.5 Must Read

Evaluated by Michael Rape 30 Mar 2009, Michael Ehlers 01 Apr 2009, Angel Nebreda 22 Oct 2009

33. Yang $Y, \operatorname{Kim} A H$, Yamada $T$, Wu B, Bilimoria PM, lkeuchi $Y$, de la Iglesia N, Shen J, Bonni A: A Cdc20-APC ubiquitin signaling pathway regulates presynaptic differentiation. Science 2009, 326:575-8.

34. van Roessel P, Elliott DA, Robinson IM, Prokop A, Brand AH: Independent regulation of synaptic size and activity by the anaphase-promoting complex. Cell 2004, I 19:707-I8.

FI000 Factor 6.0 Must Read

Evaluated by Nils Brose 28 Feb 2005

35. Juo $P$, Kaplan JM: The anaphase-promoting complex regulates the abundance of GLR-I glutamate receptors in the ventral nerve cord of C. elegans. Curr Biol 2004, 14:2057-62.

36. Herrero-Mendez A, Almeida A, Fernandez E, Maestre C, Moncada S, Bolanos JP: The bioenergetic and antioxidant status of neurons is controlled by continuous degradation of a key glycolytic enzyme by APC/C-CdhI. Nat Cell Biol 2009, I I:747-52.

FI000 Factor 3.0 Recommended

Evaluated by Valina Dawson 10 Sep 2009

37. Li M, Shin YH, Hou L, Huang X, Wei Z, Klann E, Zhang P: The adaptor protein of the anaphase promoting complex $\mathrm{Cdhl}$ is essential in maintaining replicative lifespan and in learning and memory. Nat Cell Biol 2008, 10:1083-9.

38. Garcia-Higuera I, Manchado E, Dubus P, Canamero M, Mendez J, Moreno S, Malumbres M: Genomic stability and tumour suppression by the APC/C cofactor CdhI. Nat Cell Biol 2008, 10:802-II.

F1000 Factor 3.0 Recommended

Evaluated by Maria Blasco 03 Sep 2008

39. Vescovi AL, Galli R, Reynolds BA: Brain tumour stem cells. Nat Rev Cancer 2006, 6:425-36. 